Published in final edited form as:

Neurotox Res. 2019 February ; 35(2): 421-431. doi:10.1007/s12640-018-9968-1.

\title{
Low-grade inflammation aggravates rotenone neurotoxicity and disrupts circadian clock gene expression in rats
}

\author{
Huan $\mathrm{Li}^{1,3}$, Sheng Song ${ }^{2}$, Yuan Wang ${ }^{1}$, Chun Huang ${ }^{1}$, Feng Zhang ${ }^{1}$, Jie Liu ${ }^{1,2}$, and Jau- \\ Shyong Hong ${ }^{2}$ \\ ${ }^{1}$ Key Laboratory of Basic Pharmacology of Ministry of Education and Joint International Research \\ Laboratory of Ethnomedicine of Ministry of Education, Zunyi Medical University, Zunyi, Guizhou, \\ China
}

${ }^{2}$ National Institute of Environmental Health Sciences, NIH, Research Triangle Park, NC 27709, USA

3 Jiangsu Key Laboratory of Drug Discovery for Metabolic Disease, China Pharmaceutical University, Nanjing, China, 210023

\begin{abstract}
A single injection of LPS produced low-grade neuroinflammation leading to Parkinson's disease (PD) in mice several months later. Whether such a phenomenon occurs in rats and whether such low-grade neuroinflammation would aggravate rotenone (ROT) neurotoxicity and disrupts circadian clock gene/protein expressions were examined in this study. Male rats were given two injections of LPS (2.5-7.5 mg/kg), and neuroinflammation and dopamine neuron loss were evident 3 months later. Seven months after a single LPS $(5 \mathrm{mg} / \mathrm{kg})$ injection, rats received low doses of ROT $(0.5 \mathrm{mg} / \mathrm{kg}$, sc, 5 times/week for 4 weeks) to examine low-grade neuroinflammation on ROT toxicity. LPS plus ROT produced more pronounced non-motor and motor dysfunctions than LPS or ROT alone in behavioral tests, and decreased mitochondrial complex 1 activity, together with aggravated neuroinflammation and neuron loss. The expressions of clock core genes brain and muscle Arnt-like protein-1 (Bmal1), locomotor output cycles kaput (Clock), and neuronal PAS domain protein-2 (Npas2) were decreased in LPS, ROT and LPS plus ROT groups. The expressions of circadian feedback genes Periods (Per1 and Per2) were also decreased, but Cryptochromes (Cry1 and Cry2) were unaltered. The circadian clock target genes nuclear receptor Rev-Erba (Nr1d1), and D-box-binding protein (Dbp) expressions were also decreased. Consistent with the transcript levels, circadian clock protein BMAL1, CLOCK, NR1D1 and DBP were also decreased. Thus, LPS-induced chronic low-grade neuroinflammation potentiated ROT
\end{abstract}

Corresponding authors: Jie Liu, Jie@liuonline.com;

AUTHOR CONTRIBUTIONS

Huan Li, Jie Liu, Sheng Song, Feng Zhang and John Hong conceived and designed the experiments. Huan Li, Jie Liu, Sheng Song, Yuan Wang, Chun Huang participated in the experiments performance and data analysis. Huan Li, Sheng Song, Jie Liu, Feng Zhang and John Hong wrote and revised the article. All the authors have reviewed the contents of the manuscript and approved the submitted manuscript.

Conflict of Interest Statement:

The authors declare that the research was conducted in the absence of any commercial or financial relationships that could be construed as a potential conflict of interest. 
neurotoxicity and disrupted circadian clock gene/protein expression, suggesting a role of disrupted circadian in PD development and progression.

\section{Keywords}

Parkinson's disease; lipopolysaccharide; rotenone; non-motor dysfunction; neurotoxicity; circadian clock gene expression

\section{Introduction}

Parkinson's disease (PD) is a common neurodegenerative disorder in elderly (Pringsheim et al. 2014). Accumulating evidence indicates a close association between inflammatory responses and PD (Qin et al. 2007; Stojkovska et al. 2015). Neuroinflammation is a selfdefense reaction to combat pathogen infection and typically transient and help maintain brain homeostasis. However, neuroinflammation might continue unresolved becoming persistent in pathological conditions (Block et al. 2007; Chen et al. 2016). For example, a single intraperitoneal injection of bacterial lipopolysaccharide (LPS) elicited a low-grade, long-lasting neuroinflammation leading to dopaminergic neuron loss and PD pathology several months later in mice (Qin et al. 2007, 2013; Gao et al. 2003, 2008)

Rotenone (ROT), a pesticide, has been used to produce PD animal models (Johnson and Bobrovskaya 2015). Evidence suggests that ROT facilitates DA neuron loss at least in part by induction of inflammation (Ramsey and Tansey 2012). Previous study showed that LPS and ROT interact with each other to induce toxicity in primary mesencephalic cultures (Gao et al. 2003). Intraperitoneal injection of LPS in neonate rats enhances susceptibility of nigrostriatal dopaminergic neurons to ROT neurotoxicity later in life (Cai et al. 2013). We have recently demonstrated that a single injection of LPS potentiated neurotoxicity of ROT in rats, leading to microglia activation, dopamine neuron loss in substania nigra and the decreased levels of dopamine and its metabolites in striatum (Huang et al. 2017).

It's has been well acknowledged that PD impairs both motor and non-motor functions (Sjödahl et al. 2012; Schapira et al. 2017). Most of the motor symptoms of PD could be preceded, sometimes for several years, by non-motor symptoms that include hyposomnia, sleep disorders, depression and constipation (Schapira et al. 2017). Circadian clock dysregulation is one of the important but ignored non-motor symptoms in PD (Willison et al. 2013; Videnovic et al. 2014). Circadian dysfunction has been observed in PD patients and in animal PD models including mice, rats, dogs, and non-human primates (Videnovic and Willis 2016), which may result in negative consequences to the homeostasis and even exacerbate the disease progression (Chahine et al. 2016; Li S et al. 2017; Willison et al. 2013). Circadian rhythm dysfunction is now considered as a novel environmental risk factor for PD (Lauretti et al. 2016), and a novel diagnostic and therapeutic target in PD and neurodegenerative disorders (Videnovic and Willis 2017).

Circadian clock, an endogenous timing system, is located in every cell and organ, which was regulated and maintained by transcriptional-translational feedback loops (Buhr and Takahashi 2013; Partch et al. 2014). At the core of this clock network are the transcriptional 
activators Clock, Bmal1, and Npas2, which heterodimerize CLOCK/BMAL1 and NPAS2/ BMAL1 and bind to E-box, driving transcription. Bmal1/CLOCK drives transcription of several distinct negative feedback genes, including two Cryptochrome (Cry1, Cry2) genes and three Period genes (Per1, Per2, Per3). Per and Cry proteins then heterodimerize and repress Bmal1/Clock-mediated transcription and repress their own expression. The CLOCKBMAL1 heterodimer also governs the robustly circadian expression of nuclear orphan receptors Rev-Erba (Nr1d1) and the PAR-bZip family members Dbp (Mohawk et al. 2012; Partch et al. 2014). Aberrant expression of circadian clock genes could result in dysregulated circadian rhythm at earlier stages of PD and the progression of the disease (Videnovic and Willis 2016; Schapira et al. 2017).

Circadian disruption has been reported to dysregulate inflammatory responses (CastanonCervantes et al. 2011), impair tissue homeostasis and exacerbate chronic inflammation, such as in the intestine (Pagel et al. 2017). However, little is known about circadian disruption in chronic LPS-induced neuroinflammation.

We have shown that chronic LPS exacerbated ROT-induced dopaminergic neuronal damage in adulthood rat model (Huang et al. 2017). Since the brain samples were collected in the morning from 9:00 to 11:00 am, we motivated to further examine the expression of circadian clock core genes, feedback control genes, and circadian clock-driven genes at the transcription and translation levels in this LPS combined with ROT-induced neurodegeneration in rats.

\section{Materials and methods}

\subsection{Reagents}

Lipopolysaccharide (LPS, 0111:B4) and rotenone (ROT) were purchased from Sigma Chemical Co. (St. Louis, MO, USA). Polyclonal mouse anti-Tyrosine Hydroxylase (TH) antibody (MAB318) was from MABEMD Milipore (Temecula, CA); Polyclonal rabbit anti Iba-1 antibody (019-19741) was from Wako (Richmond, VA); Antibodies against BMAL1 (ab93806), CLOCK (ab134165) were from Abcam (Cambridge, MA), antibodies against NR1D1 (ARP33733_P050) and DBP (ARP32195_P050) were from AVIVA Systems Biology (San Diego, CA). $\beta$-actin (Catalog No. mAb 4970, rabbit monoclonal) was purchased from Cell Signaling Technology (Beverly, MA). Mitochondrial Complex 1 kit was from Cominbio (Suzhou, China).

\subsection{Animals and Treatment}

Male Sprague-Dawley rats were purchased from the Experimental Animal Center of the Third Military Medical University (Chongqing, China; Certificate No. SCXK 2012-0011), and were fed rodent chow in the SPF-grade animal facilities with $21 \pm 2{ }^{\circ} \mathrm{C}$ and the light on from 8:00 to 20:00 at Zunyi Medical College. All animal experiments were performed in accordance with Chinese Guidelines of Animal Care and Welfare and the present study was approved by the Animal Care and Use Committee of Zunyi Medical University (2014-07).

To affirm LPS-induced neuroinflammation in rats, adult rats $(220 \mathrm{~g})$ were given two injections of LPS (2.5. 5.0, and $7.5 \mathrm{mg} / \mathrm{kg}, \mathrm{n}=3$ ) in continuously two days, and 3 months 
later, brains were collected, fixed in $4 \%$ paraformaldehyde to examine microglia activation via Iba1 immunostaining and dopamine neuron loss via tyrosine hydroxylase (TH) immunostaining (detailed below).

Based on this preliminary study, a single intraperitoneal injection of LPS $(5 \mathrm{mg} / \mathrm{kg})$ was used to produce low-grade neuroinflammation. Seven months later, rats received subcutaneous injection of low dose of ROT $(0.5 \mathrm{mg} / \mathrm{kg})$ or corn oil (an equivalent volume of ROT) five times a week for consecutive 4 weeks (Ojha et al., 2015; Huang et al., 2017).

After the last treatment of ROT, rats were allowed to recover for an additional 10 days before behavior tests. At the $14^{\text {th }}$ day after the last ROT treatment, brain tissues were collected (Control $=9, \mathrm{LPS}=7, \mathrm{ROT}=6, \mathrm{LPS}+\mathrm{ROT}=8$ ). To minimize the potential time-differences, all samples were collected within two hours (9:00-11:00) and one animal for each group were killed in turn to minimize the time-difference in sample collection among groups. Samples were stored $-80^{\circ} \mathrm{C}$ prior to analysis (Huang et al. 2017).

\subsection{Non-motor behavioral tests}

All behavioral data were captured by a video-monitor and analyzed using TopScan Lite 2.0 (CleverSys, Reston, VA, USA). The open-field test was used for non-motor behavioral activity (Bergamini et al. 2017). The elevated plus maze test is one of the most widely used tests for measuring anxiety-like behavior. The test is based on the natural aversion of animals for open and elevated areas, as well as on their natural spontaneous exploratory behavior in novel environments. The apparatus consists of open arms and closed arms, crossed in the middle perpendicularly to each other, and a center area (Komada et al. 2008). Y maze test was performed for learning memory. Successful alternation behavior was defined as entry into all three arms consecutively. The percentage of successful alternation was calculated as the ratio of the alternative to non-alternatives (Jin et al. 2014).

\subsection{The activity of mitochondria complex 1}

Mitochondrial complex1, also known NADH-CoQ reductase, is the largest protein complex in the inner membrane of mitochondria. Chronic systemic administration of rotenone, a specific complex 1 inhibitor, resulted in neuropathologic and behavioral changes in rats that are similar to human PD (Luo et al. 2015). Thus, the activity of mitochondria complex 1 in the brain was examined using the mitochondria complex1 assay kit (Cominbio, Suzhou, China), and results were expressed as the percentage of controls.

\subsection{Immunohistochemistry}

Rat brains were cut into $35 \mu \mathrm{m}$ sections on a horizontal sliding microtome. The free-floating sections were immunoblocked with $4 \%$ goat serum in $0.25 \%$ triton/PBS for 2 hours and then incubated with Iba-1 (1:2000), antibody overnight at $4{ }^{\circ} \mathrm{C}$. On the second day, the sections were washed by $1 \% \mathrm{BSA}$ in $0.25 \%$ triton/PBS before the incubation with anti-TH antibody (1:2000) overnight at $4{ }^{\circ} \mathrm{C}$. The double-label immunofluorescence pictures were taken under the confocal microscope by using Alexa-488 (green) and Alexa-594 (red) conjugated secondary antibodies $(1: 1,000)$ to visualize the THir or Iba-1-positive cells (Jiang et al., 2015). The number of THir and Iba-1+ cells was counted as described previously in our Lab (Liu et al., 2008). Briefly, eight evenly spaced brain slices from saline or LPS-injected rats 
were immunostained with an antibody against TH or Iba- 1 and counted. The distribution of the cell numbers from each rat was matched with the normal distribution curve to correct for errors resulting from the cutting. Samples were counted in a double-blind manner with stereology equipment. Data were expressed as percent loss compared to saline-injected controls.

\subsection{Total RNA isolation and RT-qPCR analysis}

Total RNA was extracted with TRIzol (TakaRa Biotechnology, Dalian, China). The quality and quantity of RNA were determined by Nano Drop 2000 Spectrophotometer (Thermo Scientific, Waltham, MA, USA), with 260/280 > 1.8, and by gel electrophoresis. Total RNA was reverse transcribed to cDNA with TakaRa RT kits (Dalian, China) according to manufacture instructions. The $15 \mu \mathrm{l}$ PCR reaction mix contained $3 \mu \mathrm{l}$ of cDNA $(10 \mathrm{ng} / \mathrm{ml})$, $7.5 \mu \mathrm{l}$ of $\mathrm{iQ}^{\mathrm{TM}}$ SYBR Green Supermix (Bio-Rad Laboratories, Hercules, CA), $0.5 \mu \mathrm{l}$ of primer mix (10 mM each), and $4 \mu \mathrm{l}$ of $\mathrm{dd}_{2} \mathrm{O}$. After $5 \mathrm{~min}$ denature at $95^{\circ} \mathrm{C}, 40$ cycles were performed: annealing and extension at $60^{\circ} \mathrm{C}$ for $45 \mathrm{~s}$ and denature at $95^{\circ} \mathrm{C}$ for $10 \mathrm{~s}$ in CFX96 Touch $^{\mathrm{TM}}$ Real-time PCR system (Bio-Rad Laboratories, Hercules, CA). Dissociation curve was performed after finishing 40 cycles to verify the quality of primers and amplification. The $\mathrm{Ct}$ values were used to calculate the expression of clock genes by the $2^{-\Delta \Delta \mathrm{Ct}}$ method and normalized to the housekeeping gene $\beta$-actin and GAPDH. Both $\beta$-actin and GAPDH yielded similar results, and thus the expression of interested genes was first normalized with $\beta$-actin of the same sample, and the relative transcript levels were calculated setting control as $100 \%$ as described (Li et al., 2017a; 2017b). The primers were designed with Primer3 software and listed in Table 1.

\subsection{Western Blotting Analysis}

Approximately $50 \mathrm{mg}$ cortex tissues were homogenized in $1 \mathrm{~mL}$ RIPA lysis buffer containing protease inhibitors ( $100 \mu \mathrm{M}$ phenyl methyl sulfonyl fluoride) using an electric homogenizer. Supernatant were prepared by centrifugation at $14000 \mathrm{~g}$ for $15 \mathrm{~min}$. Protein concentration was determined using a bicinchoninic acid (BCA) protein assay kit (Beyotime, China). Protein $(30 \mu \mathrm{g})$ were separated on $10 \%$ Bis-Tris Nu-PAGE gel by electrophoresis and then transferred onto polyvinylidene difluoride (PVDF) membranes. Membranes were blocked with 5\% non-fat milk and incubated over night at $4{ }^{\circ} \mathrm{C}$ with the following primary antibodies: anti- $\beta$-actin (1:5000), BMAL1 (1:1000), CLOCK (1:10000), NR1D1 (1:1000), DBP (1:1000). After thorough wash in TBST, the blots were incubated with horseradish peroxidase- conjugated secondary antibodies at 1:1000 dilutions for $2 \mathrm{~h}$. Immunoblots were visualized using chemiluminescence reagent BeyoECL Combined with (Beyotime, Shanghai, China). The image was scanned and optical density values were quantified using Adobe Photoshop CS5 software (Huang et al. 2017).

\subsection{Statistical analysis}

All data were expressed as mean \pm SEM. Comparison of multiple groups was performed using one-way ANOVA analysis followed by Social Sciences (SPSS) software (version 18.0 ), and $p<0.05$ was considered significantly different. 


\section{Results}

\subsection{LPS induced chronic neuroinflammation and dopaminergic neuron loss in substantia nigra}

Loss of dopaminergic neurons in the substantia nigra is a main character in PD patients and PD animal models (Pringsheim et al. 2014), which is associated with neuroinflammation in the area (Block et al. 2007; Chen et al. 2016). A single injection of LPS produced chronic neuroinflammation in mice (Qin et al. 2007, 2013), whether injection of LPS could also produce neuroinflammation in rats is not known. A study was therefore conducted to affirm the LPS-induced neuroinflammation in rats with three different doses. As illustrated in Figure 1, three months after injections of LPS (2.5. 5.0, and $7.5 \mathrm{mg} / \mathrm{kg}$ ) in two consecutive days, LPS induced microglia activation in a dose-dependent manner, characterized by hypertrophied morphology and intensified Iba1 staining (green). The photo of the enlarged, ramified microglia was shown in Supplemental Figure 1. Meanwhile, the numbers of microglia were increased by $6 \%, 25 \%$ and $35 \%$ after LPS $2.5,5.0$ and $7.5 \mathrm{mg} / \mathrm{kg}$, respectively. Upon microglia activation, dopamine neurons loss was evident, as evidenced by reduced THir neurons (red). The numbers of THir cells were decreased by 20\%, $43 \%$ and $55 \%$ after LPS $2.5,5.0$ and $7.5 \mathrm{mg} / \mathrm{kg}$, respectively. A whole brain image of microglia activation and THir neuron loss is shown in Supplemental Figure 2. These results demonstrated that LPS injections induced chronic neuroinflammation in rats causing delayed loss of THir neurons in substantia nigra, a brain region sensitive to LPS (Qin et al. 2007, Huang et al. 2017). However, in this study, $30 \%$ rats in the $7.5 \mathrm{mg} / \mathrm{kg}$ group died, and we have thus used $5 \mathrm{mg} / \mathrm{kg}$ LPS, the same dose as used in mice (Qin et al. 2007, 2013; Liu et al. 2008), but for longer-time periods.

\subsection{LPS exacerbated ROT produced non-motor behavioral changes}

Chronic LPS-induced low-grade neuroinflammation potentiated ROT on non-motor alternations. Fig. 2A and 2B showed the results from the open-field tests. Rats travelled (10 min) $2780 \pm 390 \mathrm{~cm}$ in control group, $2660 \pm 390 \mathrm{~cm}$ in LPS group, and $1670 \pm 360 \mathrm{~cm}$ in rotenone (ROT) group, and further decreased to $1280 \pm 320 \mathrm{~cm}$ in LPS + ROT group (Fig. $2 \mathrm{~A}$ ). The exploratory activity was also reduced in LPS + ROT group, and rats were mainly remained in local area (Fig. 2B). In the elevated combined with maze test, ROT and LPS + ROT groups showed the tendency of reduced activity in open arms (Fig. 2C). In the Y-maze teste, the correct spontaneous alternatives, a test for learning and memory, was also tended to decrease in LPS + ROT group. The values of three groups were $69 \%, 64 \%, 61 \%$, and $33 \%$ in LPS + ROT group, the percentage of spontaneous alteration was decreased from $69 \%$ to 33\% (Fig. 2D), indicating LPS + ROT could interfere with the learning and memory function, despite it was not statistically significant. Taken together with Rotarod test for motor activities (Huang et al. 2017), LPS aggravated ROT in producing behavioral deficits.

\subsection{LPS combined with ROT decreased mitochondrial function}

ROT is a well-known neurotoxicant damaging mitochondria, especially mitochondrial complex 1 (Sharma et al. 2013; Johnson and Bobrovskaya 2015). Thus, mitochondrial function was examined. As shown in Figure 3, compared with the control group, the activity 
of mitochondria complex 1 was decreased $29 \%$ in ROT group but $46 \%$ in LPS plus ROT group.

3.4. LPS combined with ROT decreased the expression of circadian clock core genes and proteins

Circadian clock core genes include Bmal1, Clock, Npas2 (Buhr and Takahashi 2013; Partch et al. 2014). Compared with the control group, the mRNA expression of Bmall decreased $11.3 \%$ in LPS group, $24.9 \%$ in ROT group, and $32.1 \%$ in LPS plus ROT group (Fig. 4A). Consisted with the mRNA expression of Bmal1 gene, the expression of BMAL1 protein was also decreased in LPS, ROT and LPS plus ROT group (the decrease was up to 29.8\%) (Fig. 4B). For mRNA expression of Clock gene, compared with the control group, its expression decreased $18.5 \%$ in LPS group, $23.1 \%$ in ROT group and $32.8 \%$ in LPS plus ROT group (Fig. 4A). Compared with the control group, CLOCK protein decreased $32.3 \%$ in ROT group and $39.1 \%$ in LPS plus ROT group (Fig. 4C). Expression of Npas 2 mRNA decreased $25.5 \%, 32.7 \%$ and $42.6 \%$ in LPS, ROT and LPS plus ROT groups, respectively. Due to the individual variation, only in LPS plus ROT group, the decrease of Npas2 was significant (Fig. 4A).

\subsection{Effects of LPS and ROT on the expression of clock feedback genes}

Circadian clock feedback genes include Per1, Per2, Cry1, and Cry2 (Buhr and Takahashi 2013; Partch et al. 2014). For Per1 gene, its expression decreased in LPS, ROT and LPS plus ROT group. The decrease was $25.6 \%$ in LPS plus ROT group that was significant. For Per2 gene, compared with the control group, Per2 expression decreased 28.7\% in LPS group, $45.1 \%$ in ROT group and 57.8\% in LPS plus ROT group, respectively. As shown in Figure 5, compared with the control group, mRNA expressions of Cry and Cry2 had no significant changes in LPS, ROT and LPS plus ROT groups.

\subsection{LPS combined with ROT decreased the expression of clock targeted genes and proteins}

Circadian clock targeted genes include Nr1d1 and Dbp (Buhr and Takahashi 2013; Partch et al. 2014). As shown in Figure 6, expressions of Nr1d1 and Dbp were decreased not only in mRNA but also in protein levels. For Nr1d1 gene, compared with the control group, its expression decreased $13.3 \%$ in LPS group, $16.6 \%$ in ROT group, and $22.4 \%$ in LPS plus ROT group (Fig. 6A). At the protein level, the expression of NR1D1 decreased in LPS, ROT and LPS plus ROT groups. Especially in LPS plus ROT group, the decrease was up to $25.9 \%$ that was significant (Fig. 6B). For Dbp gene expression, compared with the control group, its expression decreased $13.8 \%$ in LPS group, $19.0 \%$ in ROT group and $29.5 \%$ in LPS plus ROT group (Fig. 6A). At the protein level, the expression of DBP decreased in LPS, ROT and LPS plus ROT groups. Compared with the control group, DBP decreased 28.1\% in LPS plus ROT group that was significant (Fig. 6C).

\section{Discussion}

The present study clearly demonstrated that approximately 3 months after LPS injection in rats, neuroinflammation and neuron loss were evident, duplicates that observed in mice (Qin 
et al. 2007). In our prior studies, a single systemic injection of LPS aggravated ROT neurotoxicity, as evidenced by a decreased moto-activity in the Rotarod test, pathology lesions in the substantia nigra, reduced brain dopamine and dopamine metabolites, increased inflammatory mediators such as TNFa, IL-1 $1 \beta$, iNOS, and COX2, as well as the activation of p-ERK1/2, p-38, and p-JNK signaling pathways (Huang et al. 2017). The current study further demonstrated that LPS plus ROT decreased non-motor activity in Elevated Plus Maze and Open Field tests, decreased mitochondrial complex 1 activity, and importantly, disrupted the circadian clock gene expression, as evidenced by the decreased expression of circadian clock core genes (Bmal1, Clock, Npas2), feedback control genes (Per1, Per2, Cry1, Cry2), and clock target genes (Dbp, Nr1d1) were decreased at the transcriptional and/or translational levels. These novel findings highlighted the importance of circadian rhythm disruption in the pathogenesis of PD.

It is well known that PD is a chronic and progressive neurodegenerative disease exacerbating with time (Braak et al. 2004). It is becoming increasingly evident that chronic neuroinflammation is a mechanism responsible for PD (Block et al. 2007). Peripheral/ systemic inflammatory stress can also manifest in dopaminergic neuron loss likely through infiltration of peripheral leukocytes (Stojkovska et al. 2015; Gao et al. 2008). It has been reported that a single intraperitoneal LPS administration resulted in microglial activation and caused substantia nigra dopaminergic neuron loss 7 months post-injection in mice (Qin et al. 2007, 2013). This scenario in mice was duplicated in rats in the present study, another commonly used animal species in PD research, in the present study. LPS alone produced chronic neuroinflammation in SD rats in a dose-dependent manner, as evidenced by microglia activation (Iba1 staining) and dopaminergic neuron loss (TH staining) in a confocal image analysis (Figure 1). Pre-existed chronic neuroinflammation produced by a single injection of LPS in mice could aggravate other toxic effects of neurotoxicants, such as MPTP (1-methyl-4-phenyl-1,2,3,6-tetrahydropyridine) (Byler et al. 2009), a-synuclein (Zhang et al. 2018), and DSP-4 (N-(2-chloroethyl)-N-ethyl-2-bromobenzylamine), a selective noradrenergic neurotoxin (Jiang et al. 2015). The same scenario was also applied in rats, as low-grade inflammation elicited by LPS potentiated low doses of ROT in producing neurotoxicity (Huang et al. 2017).

ROT is a pesticide in the environment, and its contamination is a risk factor of PD (Brown et al. 2006; Wang et al. 2011). ROT is used as a tool to produce PD animal models (Cannon et al. 2009; Sharma et al., 2013; Johnson and Bobrovskaya 2015). The present study demonstrated that a low dose of ROT $(0.5 \mathrm{mg} / \mathrm{kg})$ decreased mitochondrial complex 1 activity in the brain by $29 \%$ but in combination of LPS by $46 \%$, fortifying that low-grade neuroinflammation from a single injection of LPS could potentiate the neurotoxic effects of ROT (Huang et al. 2017), and mitochondrial dysfunction is the major mechanism of ROT neurotoxicity.

Non-motor symptoms such as depression, constipation, sleep disorders and circadian rhythm dysfunction are frequently seen in PD patients (Tadaiesky et al. 2008; Willison et al. 2013; Schapira et al. 2017). In the present study, PD rats displayed the reduced exploratory activity and preferred to stay in local area in the open field test. In the elevated combined with maze test, PD rats preferred to stay in closed arms suggesting anxiety-like behaviors. In the Y 
maze test, the successful spontaneous alternations were significantly decreased, indicating the impaired ability of learning and memory. Thus, non-motor syndromes are important indicators for ascending nature of PD progression (Braak et al. 2004).

Circadian system has recently been proposed a novel diagnostic and therapeutic target in PD and neurodegenerative disorders (Videnovic and Willis 2016), and we have shown that repeated manganese $(\mathrm{Mn})$ administration in rats produced neurodegeneration and disruption of circadian of circadian clock genes in the brain and liver ( $\mathrm{Li}$ et al. 2017b). Circadian rhythm disruption is a major non-motor symptom in PD patients (Schapira et al. 2017; Willison et al. 2013). In ROT-induced PD rat model, circadian rhythms were disrupted (Lax et al. 2012). Circadian rhythms are controlled and maintained by a series of clock genes (Mohawk et al. 2012; Buhr and Takahashi 2013; Partch et al. 2014). Clock, Npas2 and Bmal1 are the transcriptional activators of circadian clock system, which form a heterodimer to activate the feedback control genes Per and Cry (Buhr and Takahashi 2013; Partch et al. 2014). In PD patients, sleep dysfunction seen in early Parkinson's disease may reflect a more fundamental pathology in the molecular clock underlying circadian rhythms (Breen et al. 2014; Verwey et al. 2016), and decreased expression of Bmal1 (Cai et al. 2010) and Bmal2 (Ding et al. 2011) in leukocytes of PD patients was reported. Consistent with these observations, the expression of core clock genes Bmal1 and clock was decreased in a MPTP mouse model of PD (Hayashi et al. 2013). Similarly, levels of Bmall were also decreased in rotenone model of PD (Mattam and Jagota 2015). In addition, genetic polymorphisms in Arntl (Bmal1) as well as the promoter methylation of Npas2 gene (Lin et al. 2012) were found in PD patients, implying the importance of circadian clock in PD. The present study found that expressions of mRNA and protein of Bmal1, Clock and Npas2 all were decreased in PD rat model.

The activated feedback control genes Per and Cry products accumulate, dimerize, and from a complex to interact with BMAL1-CLOCK, repressing their own transcription (Buhr and Takahashi 2013; Partch et al. 2014). In animal PD models, unilateral depletion of dopamine by 6-OHDA injection into the medial forebrain results in blunting of daily expression of the clock protein PER2 in the dorsal striatum of rats (Hood et al. 2010). In ROT-induced PD rat model, levels of Per1 and Cry1were decreased (Mattam and Jagota 2015). In a MPTPtreated mouse model of PD, expressions of Per1, Per2, Cry1 showed a decreased amplitude (Hayashi et al. 2013). Circadian rhythms of rest/activity of MitoPark mice are severely disturbed, which may be related with the loss of the midbrain dopaminergic neurons (Fifel and Cooper 2014). Importantly, rhythms in clock gene expression in the dorsal striatum are sensitive to changes in dopamine release (Verwey et al. 2016). Circadian disruption by triggering a robust neuroinflammatory reaction and degeneration of the nigral-dopaminergic neuronal system exacerbates process of PD (Lauretti et al. 2016). Consisted with PD patients and PD models, our presented study also found disrupted expressions of circadian clock feedback genes Per1 and Per2 in PD rat model, similar to our recent publication in Mninduced PD-like neurodegeneration (Li et al. 2017b).

BMAL1-CLOCK targeted genes include Nr1d1, Dbp, and many others such as ROR, Nfil3, and Tef (Buhr and Takahashi 2013; Partch et al. 2014). In a MPTP-treated mouse model of $\mathrm{PD}$, the expression of Rev-erba (Nr1d1) was significantly decreased which consistent with 
our result. Dbp is a circadian PAR-domain basic leucine zipper-transcription factor, which is controlled directly by circadian clock core genes. In addition, our previous study found dysregulation of Dbp expression also occurred in human hepatocellular carcinoma ( $\mathrm{Li}$ et al. 2017a) and in Manganism rats ( $\mathrm{Li}$ et al. 2017b). For PD animal models, the presented study first reported the dysregulation of Dbp expression. The present study demonstrated circadian clock targeted gene Nr1d1 and Dbp expressions decreased both in mRNA and protein levels. However, in Manganism rats, the mRNA expression of Nr1d1 and Dbp was increased (Li et al. 2017b). This discrepancy could be due to different models (LPS plus ROT vs Mn), and whether the increased expression of clock target genes are unique to Mn intoxication warrants further investigation.

Circadian desynchronization influences normal patterns of day-night rhythms such as sleep and alertness cycles, rest, and activity cycles (Musiek and Holtzman 2016), as observed in PD (Chahine et al. 2016; Li S et al. 2017). Circadian disruption is a common, yet mostly unrecognized, pathologic mechanism of neurodegenerative disease (Smolensky et al. 2016), and may actually drive pathogenesis early in the course of neurodegenerative diseases (Musiek and Holtzman 2016; Willison et al. 2013). Thus, an understanding of circadian clock gene dysregulation in PD not only provides a biomarker of the disease, but also could provide a chrono-therapeutic intervention target (Dallman et al. 2014; Videnovic and Willis 2016).

In summary, this study demonstrated the expression of circadian clock genes and proteins was decreased in PD rat model induced by LPS combined with ROT, implying circadian disruption contributes to PD symptoms, and is an important non-motor manifestation.

\section{Supplementary Material}

Refer to Web version on PubMed Central for supplementary material.

\section{ACKNOWLEDGMENTS}

This study was supported by the National Natural Science Foundation of China (no. 81460556). This study was supported in part by the intramural research program of National Institute of Environmental Health Sciences, NIH, USA.

\section{Reference}

Breen DP, Vuono R, Nawarathna U, Fisher K, Shneerson JM., Reddy AB, Barker RA, 2014 Sleep and circadian rhythm regulation in early Parkinson disease. JAMA Neurol. 71,589-595. [PubMed: 24687146]

Bergamini MR, Kabadayan F, Bernardi MM, Suffredini IB, Ciaramicoli MT, Kodama RM, Saraceni CH, 2017 Stress and its role in the dentin hypersensitivity in rats. Arch. Oral Biol. 73, 151-160. [PubMed: 27770641]

Block ML, Zecca L, Hong JS, 2007 Microglia-mediated neurotoxicity: uncovering the molecular mechanisms. Nat Rev Neurosci 8, 57-69. [PubMed: 17180163]

Braak H, Ghebremedhin E, Rüb U, Bratzke H, Del Tredici K, 2004 Stages in the development of Parkinson's disease-related pathology. Cell Tissue Res. 318,121-134. [PubMed: 15338272]

Brown TP, Rumsby PC, Capleton AC, Rushton L, and Levy LS, 2006 Pesticides and Parkinson's disease-is there a link? Environ Health Perspect.114, 156-164. [PubMed: 16451848] 
Buhr ED, Takahashi JS, 2013 Molecular components of the Mammalian circadian clock. Handb Exp Pharmacol. 217, 3-27.

Byler SL, Boehm GW, Karp JD, Kohman RA, Tarr AJ, Schallert T, Barth TM, 2009 Systemic lipopolysaccharide plus MPTP as a model of dopamine loss and gait instability in C57B1/6J mice. Behav Brain Res. 198, 434-439. [PubMed: 19070633]

Cannon JR, Tapias V, Na HM, Honick AS, Drolet RE, and Greenamyre JT, 2009 A highly reproducible rotenone model of Parkinson's disease. Neurobiol. Dis 34, 279-290. [PubMed: 19385059]

Cai Y, Liu S, Sothern RB, Xu S, Chan P, 2010 Expression of clock genes Per1 and Bmal1 in total leukocytes in health and Parkinson's disease. Eur J Neurol. 17, 550-554. [PubMed: 19912323]

Cai Z, Fan LW, Kaizaki A, Tien LT, Ma T, Pang Y, Lin S, Lin RC, Simpson KL, 2013 Neonatal systemic exposure to lipopolysaccharide enhances susceptibility of nigrostriatal dopaminergic neurons to rotenone neurotoxicity in later life. Dev. Neurosci 35, 155-171. [PubMed: 23446007]

Castanon-Cervantes O, Wu M, Ehlen JC, Paul K, Gamble KL, Johnson RL, Besing RC, Menaker M, Gewirtz AT, Davidson AJ, 2010 Dysregulation of inflammatory responses by chronic circadian disruption. J Immunol. 185, 5796-5805. [PubMed: 20944004]

Chahine LM, Amara AW, Videnovic A, 2017 A systematic review of the literature on disorders of sleep and wakefulness in Parkinson's disease from 2005 to 2015. Sleep Med Rev. 35, 33-50. [PubMed: 27863901]

Chen SH, Oyarzabal EA, Hong JS, 2016 Critical role of the Mac1/NOX2 pathway in mediating reactive microgliosis-generated chronic neuroinflammation and progressive neurodegeneration. Curr Opin Pharmacol. 26, 54-60. [PubMed: 26498406]

Dallmann R, Brown SA, and Gachon F, 2014 Chronopharmacology: new insights and therapeutic implications. Annu Rev Pharmacol Toxicol. 54, 339-361. [PubMed: 24160700]

Ding H, Liu S, Yuan Y, Lin Q, Chan P, Cai Y, 2011 Decreased expression of Bmal2 in patients with Parkinson's disease. Neurosci Lett. 499, 186-188. [PubMed: 21658431]

Fifel K, Cooper HM., 2014 Loss of dopamine disrupts circadian rhythms in a mouse model of Parkinson's disease. Neurobiol Dis. 71, 359-369. [PubMed: 25171792]

Gao HM, Hong JS, Zhang W, and Liu B, 2003 Synergistic dopaminergic neurotoxicity of the pesticide rotenone and inflammogen lipopolysaccharide: relevance to the etiology of Parkinson's disease. J. Neurosci 23, 1228-1236. [PubMed: 12598611]

Gao HM, and Hong JS, 2008 Why neurodegenerative diseases are progressive: uncontrolled inflammation drives disease progression. Trends Immunol 29, 357-365. [PubMed: 18599350]

Hayashi A, Matsunaga N, Okazaki H, Kakimoto K, Kimura Y, Azuma H, Ikeda E, Shiba T, Yamato M, Yamada K, Koyanagi S, Ohdo S, 2013 A disruption mechanism of the molecular clock in a MPTP mouse model of Parkinson's disease. Neuromolecular Med. 15, 238-251. [PubMed: 23292542]

Hood S, Cassidy P, Cossette MP., Weigl Y, Verwey M, Robinson B, Stewart J, Amir S, 2010 Endogenous dopamine regulates the rhythm of expression of the clock protein PER2 in the rat dorsal striatum via daily activation of D2 dopamine receptors. J Neurosci. 30,14046-14058. [PubMed: 20962226]

Huang C, Zhu L, Li H, Shi FG, Wang GQ, Wei YZ, Liu J, Zhang F, 2017 Adulthood Exposure to Lipopolysaccharide Exacerbates the Neurotoxic and Inflammatory Effects of Rotenone in the Substantia Nigra. Front Mol Neurosci. 10:131. [PubMed: 28533741]

Jiang L, Chen SH, Chu CH, Wang SJ, Oyarzabal E, Wilson B, Sanders V, Xie K, Wang Q, Hong JS, 2015 A novel role of microglial NADPH oxidase in mediating extra-synaptic function of norepinephrine in regulating brain immune homeostasis. Glia. 63, 1057-1072. [PubMed: 25740080]

Jin F, Gong QH., Xu YS, Wang LN, Jin H, Li F, Li LS, Ma YM, Shi JS, 2014 Icariin, a phosphodiesterase-5 inhibitor, improves learning and memory in APP/PS1 transgenic mice by stimulation of NO/cGMP signaling. Int J Neuropsychopharmacol. 17,871-881. [PubMed: 24513083]

Johnson ME, Bobrovskaya L 2015 An update on the rotenone models of Parkinson's disease: their ability to reproduce the features of clinical disease and model gene-environment interactions. Neurotoxicology. 46, 101-116. [PubMed: 25514659] 
Komada M, Takao K, Miyakawa T, 2008 Elevated plus maze for mice. J Vis Exp. 22, pii: 1088, doi: 10.3791/1088.

Lax P, Esquiva G, Esteve-Rudd J, Otalora BB, Madrid JA, Cuenca N, 2012 Circadian dysfunction in a rotenone-induced parkinsonian rodent model. Chronobiol Int. 29, 147-156. [PubMed: 22324553]

Lauretti E, Di, Meco A, Merali S, Praticò D, 2016 Circadian rhythm dysfunction: a novel environmental risk factor for Parkinson's disease. Mol Psychiatry. 22, 280-286. [PubMed: 27046648]

Li H, Lu YF, Chen H, Liu J, 2017a Dysregulation of metallothionein and circadian genes in human hepatocellular carcinoma. Chronobiol Int. 34, 192-202. [PubMed: 27997226]

Li H, Fan X, Luo Y, Song S, Liu J, Fan Q, 2017b Repeated manganese administration produced abnormal expression of circadian clock genes in the hypothalamus and liver of rats. Neurotoxicology 62, 39-45. [PubMed: 28511934]

Li S, Wang Y, Wang F, Hu LF, Liu CF, 2017 A New Perspective for Parkinson's Disease: Circadian Rhythm. Neurosci Bull. 33,62-72. [PubMed: 27995565]

Liu Y, Qin L, Wilson B, Wu X, Qian L, Granholm AC, Crews FT, Hong JS, 2008 Endotoxin induces a delayed loss of TH-IR neurons in substantia nigra and motor behavioral deficits. Neurotoxicology 29, 864-870. [PubMed: 18471886]

Lin Q, Ding H, Zheng Z, Gu Z, Ma J, Chen L, Chan P, Cai Y, 2012 Promoter methylation analysis of seven clock genes in Parkinson's disease. Neurosci Lett. 507, 147-150. [PubMed: 22193177]

Luo Y, Hoffer A, Hoffer B, Qi X, 2015 Mitochondria: A Therapeutic Target for Parkinson's Disease? Int J Mol Sci. 16, 20704-20730. [PubMed: 26340618]

Mattam U, Jagota A, 2015 Daily rhythms of serotonin metabolism and the expression of clock genes in suprachiasmatic nucleus of rotenone-induced Parkinson's disease male Wistar rat model and effect of melatonin administration. Biogerontology 16,109-123. [PubMed: 25430725]

Mohawk JA, Green CB, Takahashi JS, 2012 Central and peripheral circadian clocks in mammals. Ann. Rev. Neurosci 35, 445-462. [PubMed: 22483041]

Musiek ES, Holtzman DM, 2016 Mechanisms linking circadian clocks, sleep, and neurodegeneration. Science 354, 1004-1008. [PubMed: 27885006]

Ojha S, Javed H, Azimullah S, Abul Khair SB, Haque ME, 2015 Neuroprotective potential of ferulic acid in the rotenone model of Parkinson's disease. Drug Des. Devel. Ther 9, 5499-5510.

Pagel R, Bär F, Schröder T, Sünderhauf A, Künstner A, Ibrahim SM, Autenrieth SE, Kalies K, König P, Tsang AH, Bettenworth D, Divanovic S, Lehnert H, Fellermann K, Oster H, Derer S, Sina C, 2017 Circadian rhythm disruption impairs tissue homeostasis and exacerbates chronic inflammation in the intestine. FASEB J. 2017 31(11):4707-4719.

Partch CL, Green CB, Takahashi JS, 2014 Molecular architecture of the mammalian circadian clock. Trends Cell Biol. 24:90-99. [PubMed: 23916625]

Pringsheim T, Jette N, Frolkis A, Steeves TD, 2014 The prevalence of Parkinson's disease: a systematic review and meta-analysis. Mov Disord. 29, 1583-1590. [PubMed: 24976103]

Qin L, Wu X, Block ML, Liu Y, Breese GR, Hong JS, Knapp DJ, Crews FT, 2007 Systemic LPS causes chronic neuroinflammation and progressive neurodegeneration. Glia 55, 453-462. [PubMed: 17203472]

Qin L, Liu Y, Hong JS, Crews FT, 2013 NADPH oxidase and aging drive microglial activation, oxidative stress, and dopaminergic neurodegeneration following systemic LPS administration. Glia 61, 855-868. [PubMed: 23536230]

Ramsey CP, Tansey MG, 2014 A survey from 2012 of evidence for the role of neuroinflammation in neurotoxin animal models of Parkinson's disease and potential molecular targets. Exp Neurol. 256, 126-132. [PubMed: 23726958]

Sharma S, Moon CS, Khogali A, Haidous A, Chabenne A, Ojo C, Jelebinkov M, Kurdi Y, Ebadi M, 2013 Biomarkers in Parkinson's disease (recent update). Neurochem Int. 63, 201-229. [PubMed: 23791710]

Schapira AHV, Chaudhuri KR, Jenner P, 2017 Non-motor features of Parkinson disease. Nat Rev Neurosci. 18(8):509. [PubMed: 28720825]

Stojkovska I, Wagner BM., Morrison BE, 2015 Parkinson's disease and enhanced inflammatory response. Exp. Biol. Med. (Maywood). 240, 1387-1395. [PubMed: 25769314] 
Sjödahl Hammarlund. C., Hagell P, Nilsson MH, 2012 Motor and non-motor predictors of illnessrelated distress in Parkinson's disease. Parkinsonism Relat Disord. 18, 299-302. [PubMed: 22100143]

Smolensky MH., Hermida RC, Reinberg A, Sackett-Lundeen L, Portaluppi F, 2016 Circadian disruption: New clinical perspective of disease pathology and basis for chronotherapeutic intervention. Chronobiol Int. 33, 1101-1119. [PubMed: 27308960]

Tadaiesky MT, Dombrowski PA, Figueiredo CP, Cargnin-Ferreira E, Da Cunha C., Takahashi RN, 2008 Emotional, cognitive and neurochemical alterations in a premotor stage model of Parkinson's disease. Neuroscience 156, 830-840. [PubMed: 18817851]

Videnovic A, Willis GL, 2016 Circadian system - A novel diagnostic and therapeutic target in Parkinson's disease? Mov Disord. 31, 260-269. [PubMed: 26826022]

Videnovic A, Laza r A.S., Barker RA, Overeem S, 2014 The clocks that time us'--circadian rhythms in neurodegenerative disorders. Nat Rev Neurol. 10, 683-693. [PubMed: 25385339]

Verwey M, Dhir S, Amir S, 2016 Circadian influences on dopamine circuits of the brain: regulation of striatal rhythms of clock gene expression and implications for psychopathology and disease. F1000Res. pii: F1000 Faculty Rev-2062.

Wang A, Costello S, Cockburn M, Zhang X, Bronstein J, Ritz B, 2011 Parkinson's disease risk from ambient exposure to pesticides. Eur. J. Epidemiol 26, 547-555. [PubMed: 21505849]

Willison LD, Kudo T, Loh DH, Kuljis D, Colwell CS, 2013 Circadian dysfunction may be a key component of the non-motor symptoms of Parkinson's disease: insights from a transgenic mouse model. Exp Neurol. 243, 57-66. [PubMed: 23353924]

Zhang W, Gao JH, Yan ZF, Huang XY, Guo P, Sun L, Liu Z, Hu Y, Zuo LJ, Yu SY, Cao CJ Wang XM, Hong JS, 2018 Minimally toxic dose of lipopolysaccharide and alpha-Synuclein oligomer elicit synergistic dopaminergic neurodegeneration: Role and mechanism of microglial NOX2 activation. Mol Neurobiol. 55, 619-632. [PubMed: 27975175] 


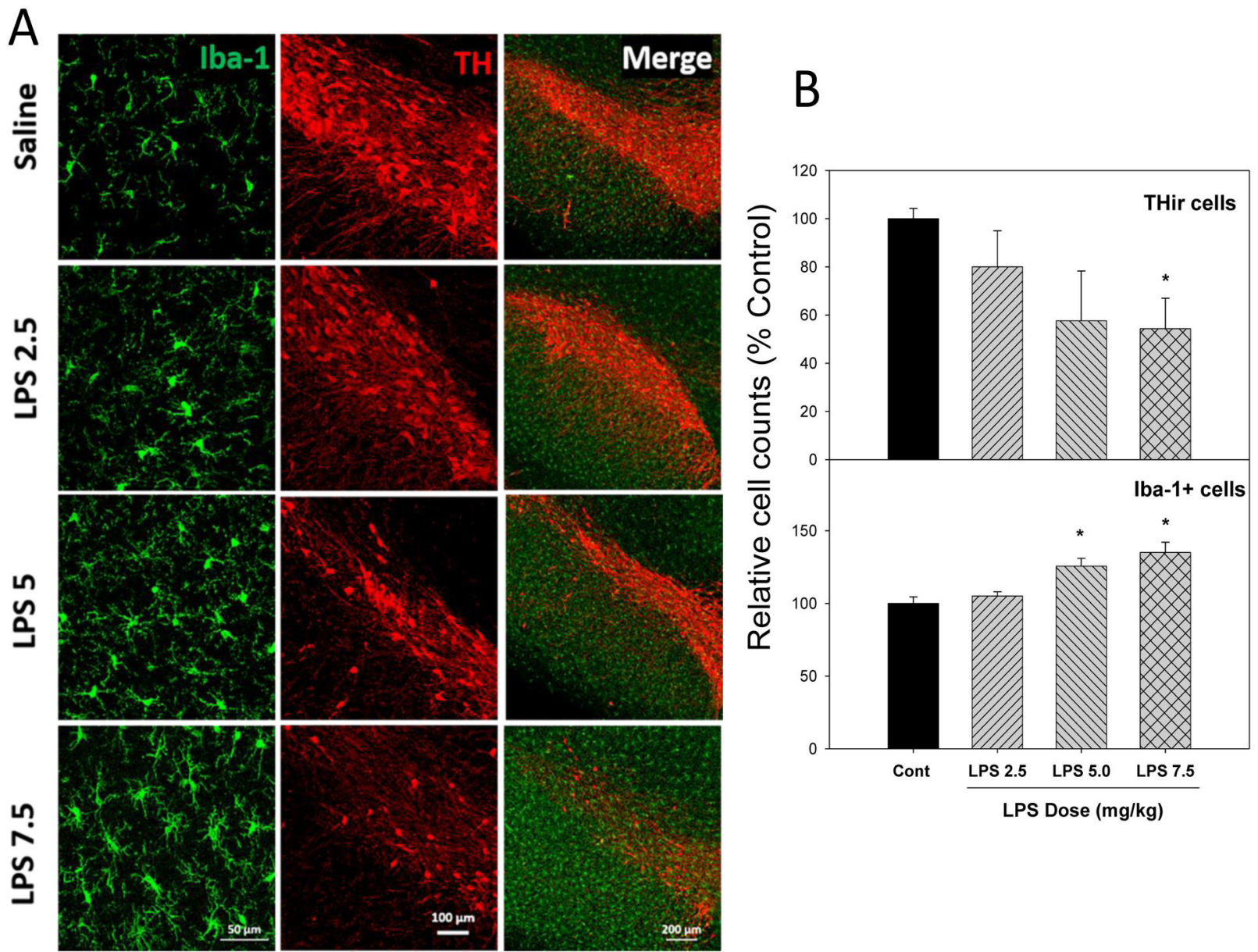

Figure 1.

LPS induced chronic neuroinflammation in rats. Adult male SD rats were given injections of saline, LPS 2.5, 5, and $7.5 \mathrm{mg} / \mathrm{kg}$, ip, and 3 months later, the brains were collected for double stain for dopaminergic neurons (TH-strain, red) and microglia (Iba1-stain, green) (A), and their quantitative cell counts were expressed as $\%$ of Controls as described previously (Liu et al. 2008) (B). Values are the mean \pm SEM $(n=3)$.

$*$ Significantly different from the control group, $\mathrm{p}<0.05$. 

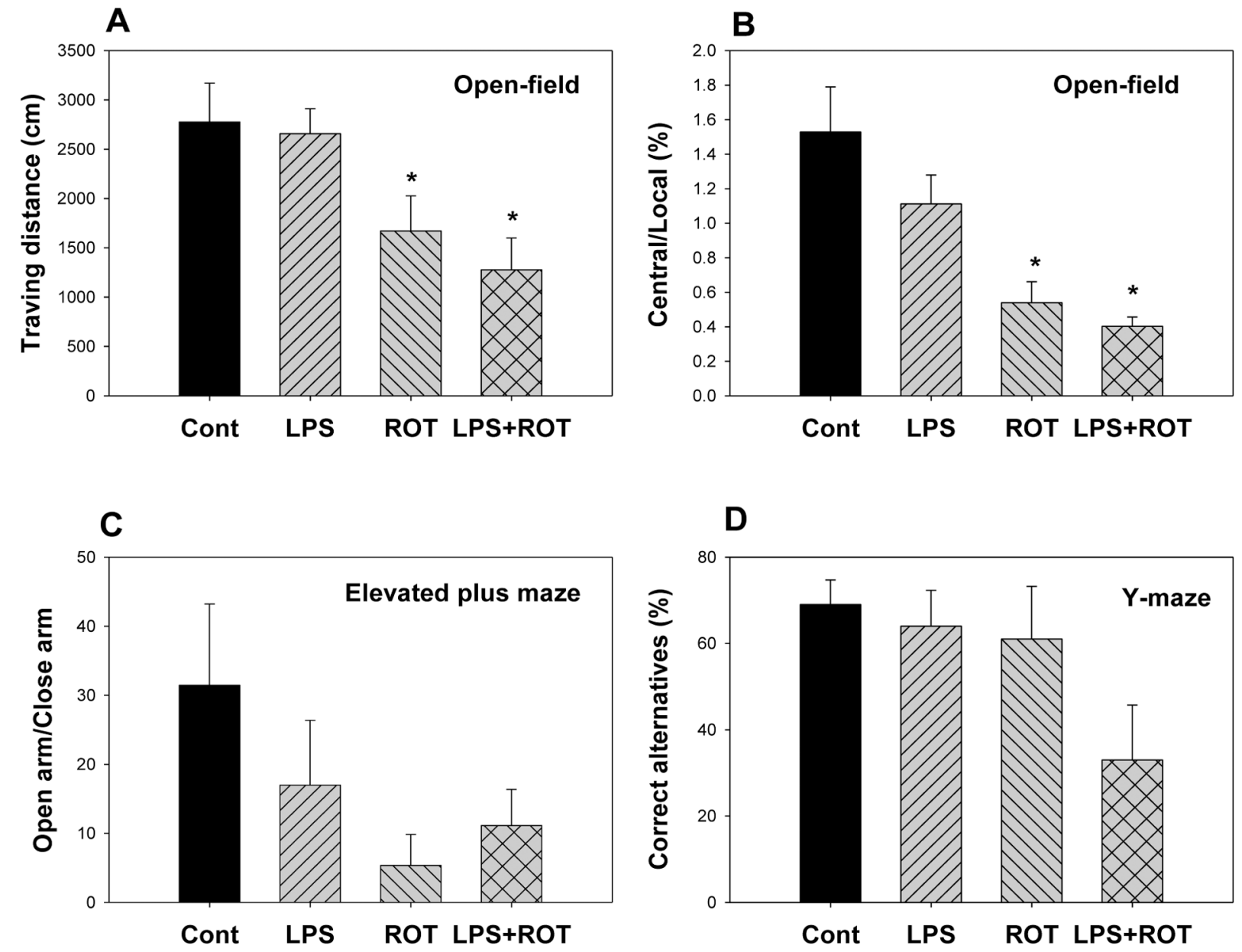

Figure 2.

Effects of LPS combined with ROT on non-motor behavior. Rats were given LPS ( $5 \mathrm{mg} / \mathrm{kg}$, i.p. $\mathrm{x} 1)$, and 7 months later received 20 injections of rotenone $(0.5 \mathrm{mg} / \mathrm{kg}$, s.c., 5 time/week for 4 weeks), and the tests was performed 10 days after the last dose of rotenone. A, Openfield traveling distance; B, Open-field central/local activity ration; C. Open arm/closed arm in elevated combined with maze test; D. Correct alternative rate in the Y maze test. Values are the mean \pm SEM $(n=6-9)$.

*Significantly different from the control group, $\mathrm{p}<0.05$. 


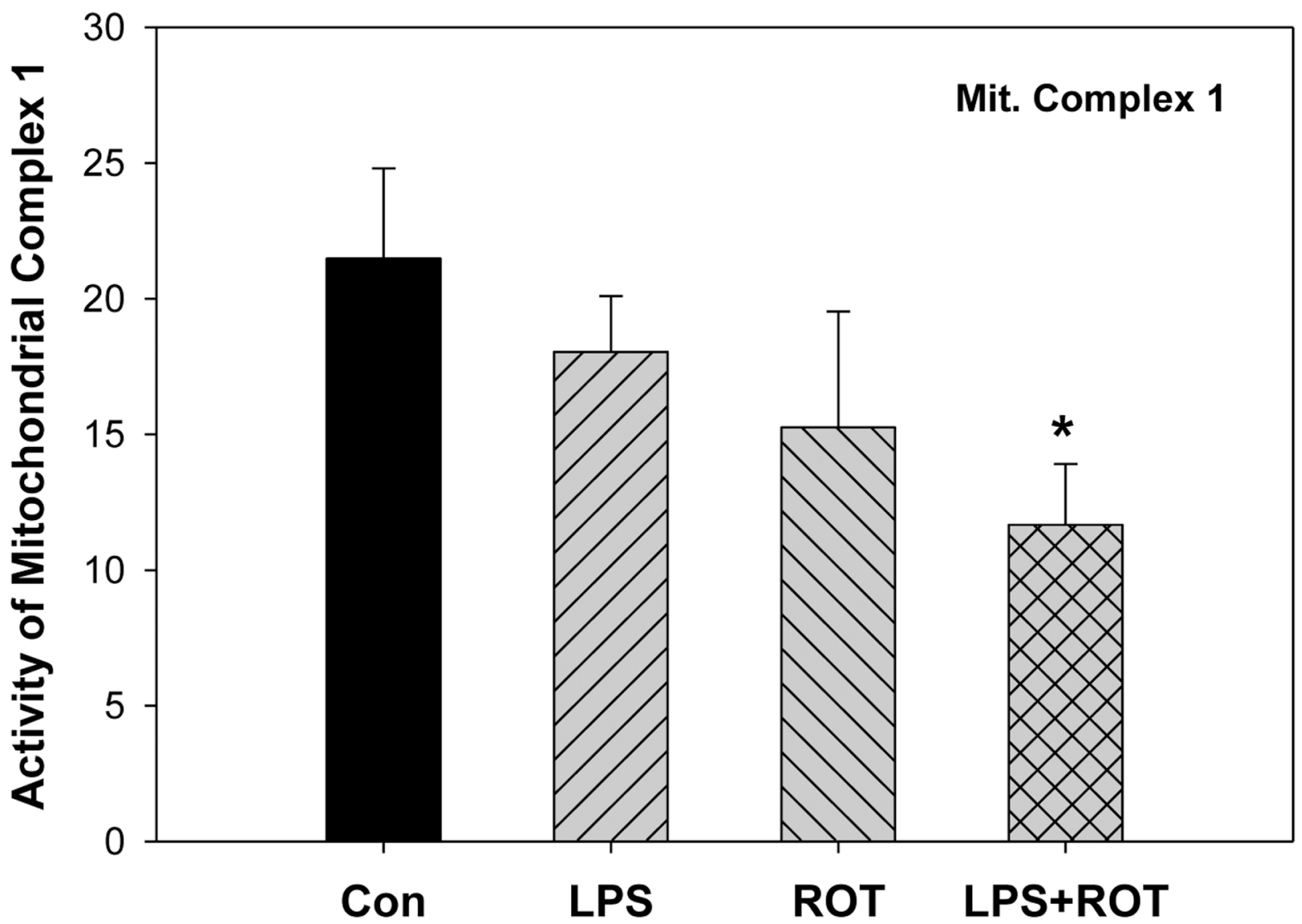

Figure 3.

Effects of LPS and ROT on the activity of mitochondria complex 1 in cortex. Values are expressed as the percentage of controls, mean \pm SEM $(n=6-9)$.

$*$ Significantly different from the control group, $\mathrm{p}<0.05$. 
A

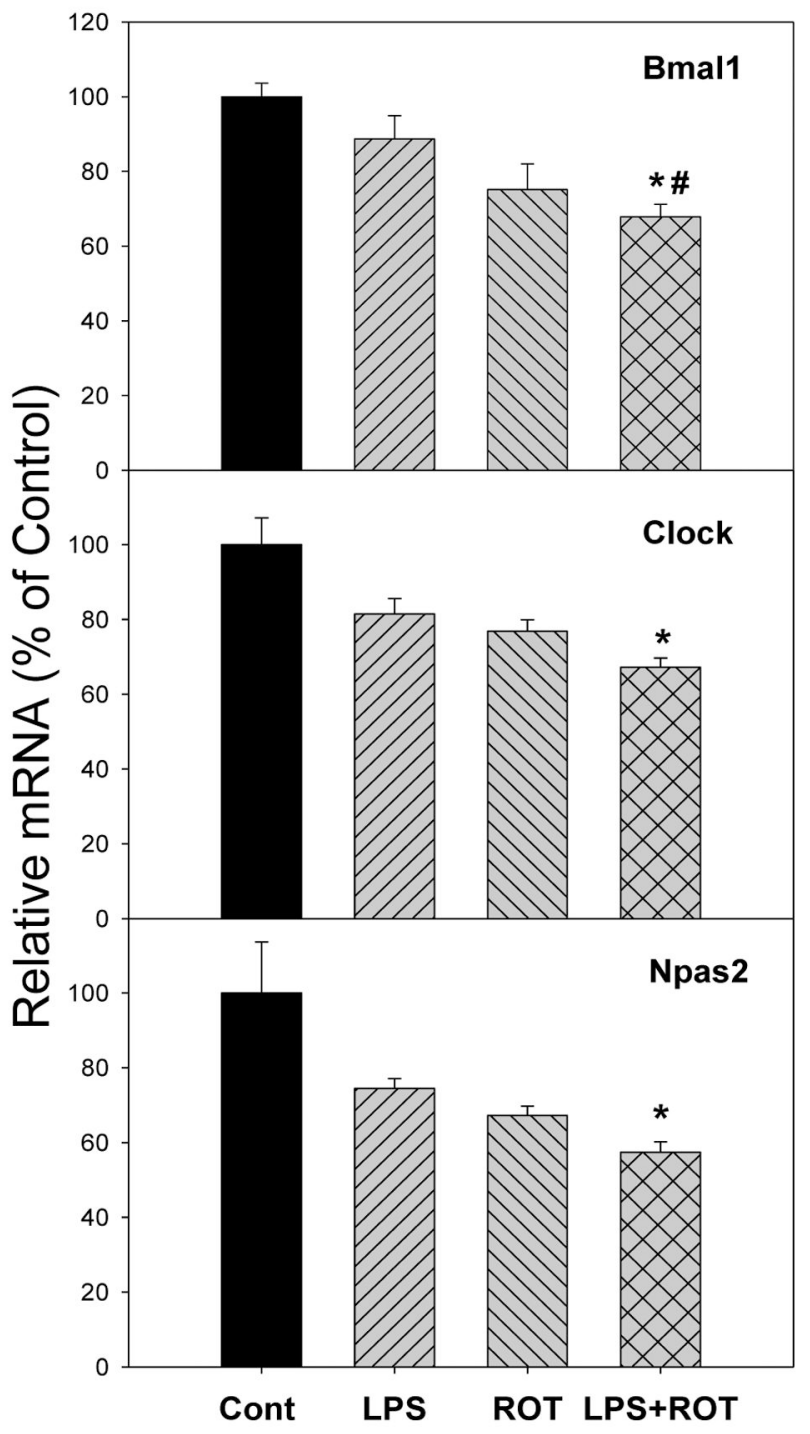

B
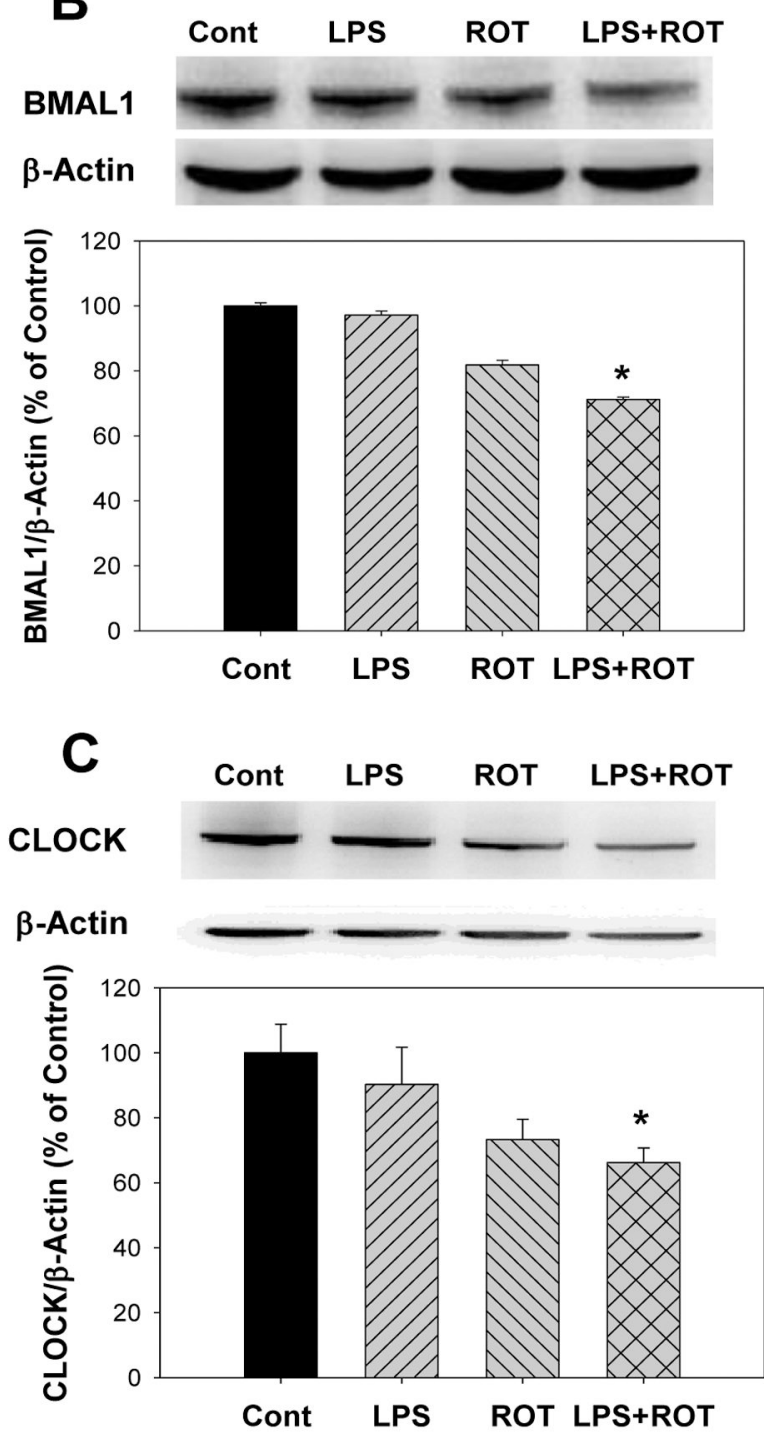

Figure 4.

Effects of LPS and ROT on the expression of circadian clock core genes and proteins. Rats were given LPS ( $5 \mathrm{mg} / \mathrm{kg}$, i.p. x1), and 7 months later 20 injections of rotenone $(0.5 \mathrm{mg} / \mathrm{kg}$, s.c., 5 time/week for 4 weeks). Two weeks after the last ROT treatment, rats were sacrificed and cortex was collected for analysis. A, mRNA expression of circadian clock core genes; B, The expression of BMAL1 protein; C, The expression of CLOCK protein. Values were mean \pm SEM $(n=6-9)$.

*Significantly different from the control group,

\#Significantly different from the LPS group, $\mathrm{p}<0.05$. 


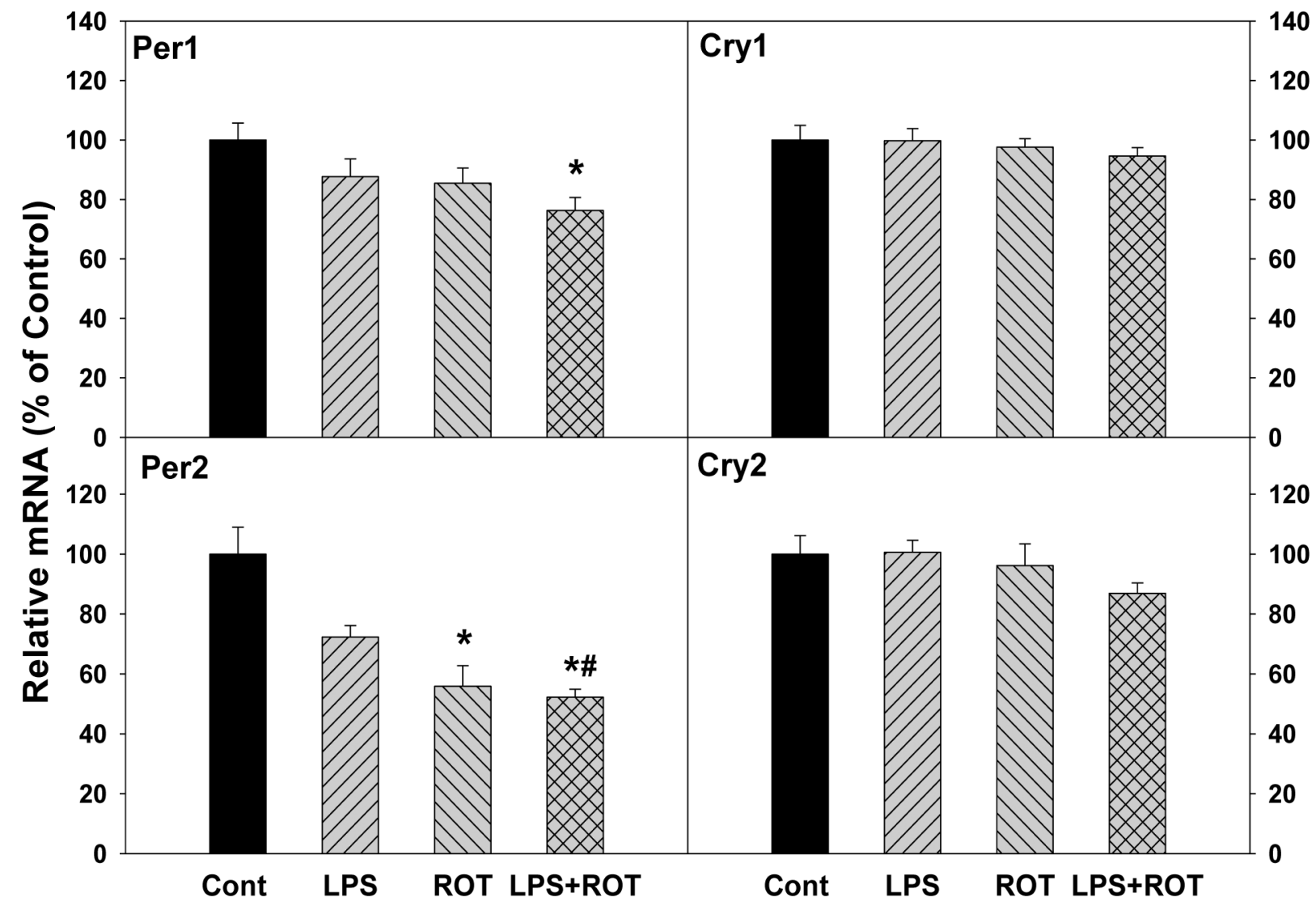

Figure 5.

Effects of LPS and ROT on the expressions of circadian clock feedback gene Per1, Per2, Cry1, and Cry2. Rats were given LPS ( $5 \mathrm{mg} / \mathrm{kg}$, i.p. x1), and 7 months later 20 injections of rotenone $(0.5 \mathrm{mg} / \mathrm{kg}$, s.c., 5 time/week for 4 weeks $)$. Two weeks after the last ROT treatment, rats were sacrificed and cortex was collected for analysis. Values are mean \pm SEM $(\mathrm{n}=6-9)$.

*Significantly different from the control group,

\#Significantly different from the LPS group, $\mathrm{p}<0.05$. 

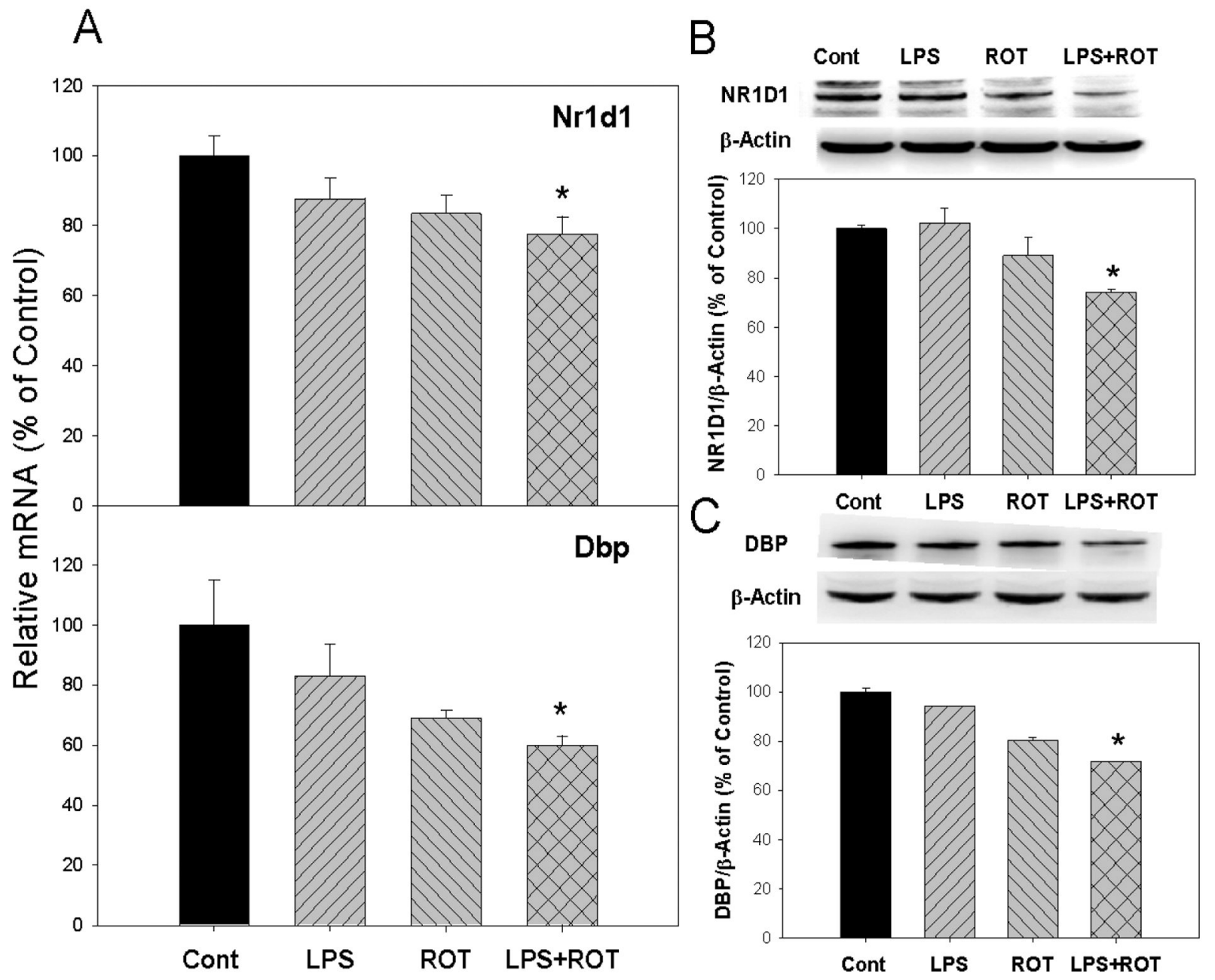

Figure 6.

Effects of LPS and ROT on the expression of circadian clock target genes. Rats were given LPS ( $5 \mathrm{mg} / \mathrm{kg}$, i.p. x1), and 7 months later 20 injections of rotenone $(0.5 \mathrm{mg} / \mathrm{kg}$, s.c., 5 time/ week for 4 weeks). Two weeks after the last ROT treatment, rats were sacrificed and cortex was collected for analysis. A, mRNA expression of circadian clock target gene Nr1d1 and Dbp; B, the expression of NR1D1 protein; and C, the expression of DBP protein. Values are mean \pm SEM $(n=6-9)$.

$*$ Significantly different from the control group $\mathrm{p}<0.05$. 
Table 1.

Primer sequences for rat circadian clock genes

\begin{tabular}{llll}
\hline Gene & Accession & Forward & Reverse \\
\hline p-actin & NM-031144 & AGCCATGTACGTAGCCATCC & ACCCTCATAGATGGGCACAG \\
Bmal1 & NM_024362 & AGCCAGTGAACGGGGAAATA & TATGCCAAAATAGCCGTCGC \\
Clock & NM_021856 & CTGTTACATCAGCACGCCTC & TACTGCTTGGCTCTTCTGCT \\
Npas2 & NM_001108214 & CCTAGCCCCTCCTGTAATGG & AGGTTCGTCAGCTACACACA \\
Cry1 & NM_198750 & ACGTGATAGGGAAGTGCACA & GTGCCTCAGTTTCTCCTCCT \\
Per1 & NM_001034125 & AAACAACAGCCACGGTTCTC & CACAAGCCGTTACATCGAGG \\
Per2 & NM_031678 & ACAGCTACACCACCCCTTAC & CAACACTGACACGGCAGAAA \\
Nr1d1 & NM_001113422 & AGCTGGTGAAGACATGACGA & TGCCATTGGAGCTGTCACTA \\
Dbp & NM_012543 & GCGAGAAGTGCAAAATTGGC & CTATAGTCTGGGCGAGTGGG \\
\hline
\end{tabular}

Neurotox Res. Author manuscript; available in PMC 2019 May 31. 